\title{
Autonomous Synthesis of Fluorescent Silica Biodots Using Engineered Fusion Proteins
}

\author{
Tolga T. Olmez, ${ }^{\dagger}$ Esra Yuca, ${ }^{\dagger, \S}$ Erol Eyupoglu, ${ }^{\ddagger}$ Hazal B. Catalak, ${ }^{\dagger}$ Ozgur Sahin, ${ }^{\dagger,}$
} and Urartu Ozgur Safak Seker*,†

${ }^{\dagger}$ UNAM-National Nanotechnology Research Center, Institute of Materials Science and Nanotechnology, and ${ }^{\ddagger}$ Department of Molecular Biology and Genetics, Faculty of Science, Bilkent University, Ankara 06800, Turkey

${ }^{\S}$ Department of Molecular Biology and Genetics, Faculty of Arts and Science, Yildiz Technical University, Istanbul 34210, Turkey

\section{Supporting Information}

\begin{abstract}
Formation of biological materials is a well-controlled process that is orchestrated by biomolecules such as proteins. Proteins can control the nucleation and mineralization of biomaterials, thereby forming the hard tissues of biological organisms, such as bones, teeth, and shells. In this study, the design and implementation of multifunctional designer proteins are demonstrated for fluorescent silica micro/nanoparticle synthesis. The R5 motif of silaffin polypeptide, which is known for its silicification capability, was fused genetically into three spectrally distinct fluorescent proteins with the intention of forming modified fluorescent proteins. The bifunctional R5 peptide domain served as a tag to provide silica synthesis at ambient conditions. Three functional fusion constructs have been prepared, including GFPmut3-R5, Venus YFP-R5, and mCherry-R5. Recombinant fluorescent proteins were purified using silica-binding peptide tag through silica gel resin. Purified proteins were tested for their binding affinity to silica using quartz crystal microbalance with dissipation monitoring to make sure they can interact strong enough with the silica surfaces. Later, engineered fluorescent proteins were used to synthesize silica nano/microparticles using silica precursor materials. Synthesized silica particles were investigated for their fluorescence properties, including time-resolved fluorescence. Additionally, elemental analysis of the particles was carried out using electron energy loss spectroscopy and energy-filtered transmission electron microscopy. Last, they were tested for their biocompatibility. In this study, we aimed to provide a biomimetic route to synthesize fluorescent silica nanoparticles. Recombinant fluorescent proteins-directed silica nanoparticles synthesis offers a onestep, reliable method to produce fluorescent particles both for biomaterial applications and other nanotechnology applications.
\end{abstract}

\section{INTRODUCTION}

Biological synthesis and assembly of inorganic solid nanostructures are performed by most organisms in nature. ${ }^{1,2}$ Many organisms are capable of synthesizing materials to form hard tissues, such as bones, teeth, and shells. ${ }^{3}$ These structures are synthesized by controlling many different protein cascades. For instance, biological apatite crystals possess well-defined elemental compositions and crystal structures that are responsible for imparting hard tissues such as bone and teeth with their characteristic properties. Extracellular matrix proteins regulate the nucleation and growth of these biological apatites during hard tissue development. ${ }^{4-6}$ The formation of the layered shell structure of mollusks is also regulated by peptides and proteins. Inorganic $\mathrm{CaCO}_{3}$ and organic biopolymers provide the toughness and fracture strength of nacre. ${ }^{7,8}$ Diatoms are microscopic unicellular algae that can synthesize silica nanostructures around the cell membrane to keep themselves protected from external threats. Silica cell walls (frustules) of diatoms have intriguing shapes and enhanced optical properties that help in the light harvesting process of photosynthesis. Newly divided diatom cells retrieve half of the old frustule from the mother cell, whereas the other half is produced from silica precursors. ' Frustules are composed of hierarchical nanostructured species-specific patterns. Frustule structures have been shown to enhance the conversion of light to chemical energy by increasing the interaction of photons with light harvesting molecules and focusing light onto chloroplasts. $^{10,11}$

Although well-established chemical and physical methods exist for nano/micromaterial synthesis, ${ }^{12}$ these approaches are often obliged to use environmentally harmful substances in greater amounts and reactions generally take place in extreme conditions in terms of the solution $\mathrm{pH}$, humidity, environmental pressure, and temperature. On the other hand, biological synthesis generally happens at ambient conditions and involves harmless substances in minute quantities, which is better for the environment. ${ }^{13,14}$ Until this time, mimicking of frustules by biological synthesis methods has been limited largely to the replication of processes that have already been achieved by chemical and physical methods. Living biohybrids refer to systems involving the encapsulation of yeast or bacterial cells that maintain their metabolic activity, as well as conceptual

Received: November 10, 2017

Accepted: January 3, 2018

Published: January 18, 2018 


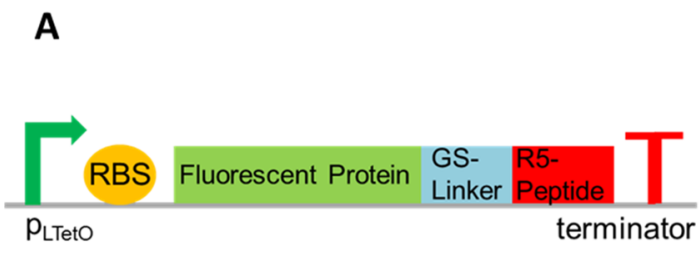

C
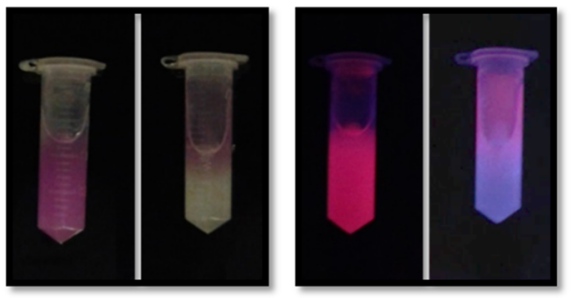

D
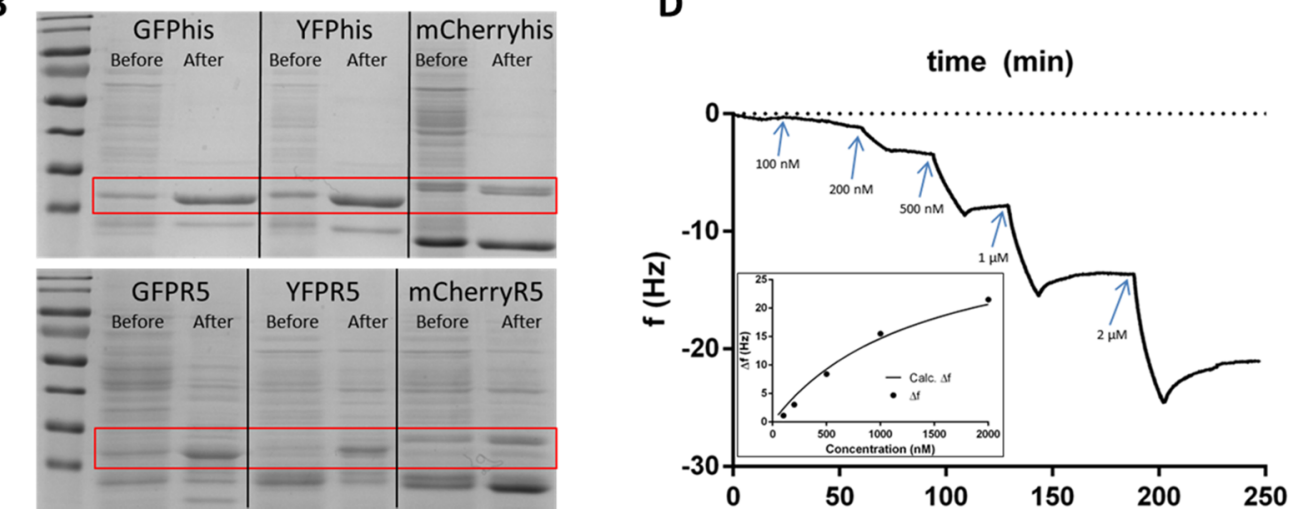

Figure 1. Plasmid design map (A) for fluorescent protein/R5 peptide fusion proteins. pLtetO: tetracycline promoter, ribosome binding site, FPs: fluorescent proteins (GFPmut3, Venus YFP, and mCherry RFP) R5: silaffin R5 peptide rrnB-T1: terminator sequence. Sodium dodecyl sulfatepolyacrylamide gel electrophoresis (SDS-PAGE) analysis (B) before and after purification using histidine tag (upper gel) and silica tag (lower gel). Before: whole cell extraction of yellow fluorescent protein (YFP)-R5 protein from bacteria, after: elution of fusion proteins by either histidine or silica-binding resin. Expected bands for the fusion proteins were highlighted in red squares. Light (left) and fluorescence (right) images (C) of purified mCherry-R5 protein from silica gel. Images on the left of each panel show the binding of mCherry-R5 isolates to the silica resin, whereas those on the right show the after-elution condition. (D) Quartz crystal microbalance (QCM) measurement of YFP-R5. Binding kinetics to the silica quartz surface is shown as a resonance frequency change. Protein solutions in phosphate-buffered saline (PBS) are sequentially administered in increasing concentrations (shown by arrows). Quartz crystal microbalance with dissipation monitoring (QCM-D) experiments ran for other protein constructs too, adsorption isotherms can be found in Supporting Information.

whole cell biosynthesizers that can perform biomineralization at ambient conditions. ${ }^{15,16}$ However, bottom-up biosynthesis of complex, hierarchical silica nanostructures depends on the development of strict temporal and spatial control on a genetic multi-input biosystem.

Although frustules are composed almost entirely of silica, silicifying peptides and polysaccharides contribute to the threedimensional (3D) organization of silica. ${ }^{17,18}$ Among diatom silica cell wall-related proteins, silaffins are central as a template for the synthesis and hierarchical order of frustules. R5 peptide is a well-studied subunit of the silaffin protein of Cylindrotheca fusiformis and has been shown to induce silica structure formation from silica precursors like silicic acid. ${ }^{19}$ Studies also show that bacteria-synthesized R5 peptide can induce the synthesis of silica nanoparticles from precursor molecules. ${ }^{20}$ Several studies have demonstrated the potency of R5 peptide in synthesizing silica nano/microstructures capable of encapsulating protein cargo. ${ }^{21-24}$ Additionally, fusion of R5 with green fluorescent protein (GFP) was shown to induce the formation of silica nanoparticles while allowing protein purification by histidine tagging or S-tagging. ${ }^{25,26}$

Fluorescent nanoparticles have been of great interest for many applications because of their unusual stability and optical properties (such as narrow emission spectrum etc.). Among the fluorescent nanoparticles, quantum dots have been investigated and developed for many applications. They have excellent optical properties that are useful for applications in physics, but a major drawback for their use in humans is their inherent toxicity. ${ }^{27,28}$ Therefore, development of biocompatible fluo- rescent particles having optical properties that are comparable to quantum dots is of great importance for applications in biomedicine. When fused to a fluorescent protein (FP), R5 can nucleate silica around the FP in a manner analogous to the formation of diatom frustules. The resulting hybrid dots can increase the optical properties of FPs and serve as a candidate for the development of optically superior fluorescent modules exhibiting low toxicity profiles, which is of substantial importance for their biomedical applications.

Affinity tags have found much use in biology to recover proteins from whole cell extracts in high purity. ${ }^{29}$ Rapid and cost-effective methods for protein affinity purification have been developed using silica tags. ${ }^{30,31}$ Silica tags such as ribosomal protein L2, CotB1p, and Car9 are utilized as silicainteracting molecules and show affinity for silica gel matrices or surfaces due to their positively charged ( $\mathrm{R}$ or K-rich) compositions. $^{32}$ The majority of affinity tags must be cleaved by enzymes from the target protein to ensure the latter's function, avoid immune response, or allow crystallization. ${ }^{33}$ However, tag removal causes an increase in the total cost and prolongs the process.

In this study, to further increase the potency of silica tags in silica biohybrid synthesis studies, R5 peptide is used as a fusion tag for fluorescent proteins (FPs) prior to the synthesis process, enabling both the purification of the fusion protein in a silica gel matrix and formation of silica particles from precursor molecules. Final particle silica entrapment does not affect the fluorescent lifetime of the synthesized fluorescent proteins and they are found to be biocompatible. 


\section{RESULTS AND DISCUSSION}

2.1. Genetic Constructs and Heterologous Expression of FPs. The position of the R5 tag may dramatically change the function of fluorescent proteins. To test whether this effect altered peptide function under the present design, R5 tagcoding sequence was placed on either the $5^{\prime}$ or the $3^{\prime}$ end of the genetic constructs. Although fluorescence microscopy results have given positive green fluorescence for both of the constructs, fluorescence spectroscopy results reveal that the excitation-emission maxima shifted for R5-GFP, where the silica tag-coding sequence was added to the upstream of the GFP-coding sequence (Figure S1). To avoid excitationemission shifts, the remaining constructs were designed to attach the R5 tag-coding sequence to the $3^{\prime}$ end of the fluorescent protein-coding sequence in all genetic designs (Figure 1A). Two different approaches were included while adding R5 tag-coding sequence to the end of each fluorescent protein DNA sequence. The first approach involves the addition of the $\mathrm{R} 5$ tag-coding sequence by successive extension polymerase chain reactions (PCRs). We also synthesized the R5 tag DNA sequence to use it as one of the fragments for the Gibson assembly reaction. Both approaches were proven to be successful by Sanger sequencing (.A). Constructs were ligated into an aTc-inducible vector. The inducible vector also includes a tightly regulated synthetic riboregulator system (taRNAcrRNA) to inhibit possible leakage. ${ }^{34}$ The genetic system leaks very little or not at all when the inducer is absent (Figures S3 and S4).

Escherichia coli (E. coli) cells that are transformed with GFPR5, R5-GFP, mCherry-R5, and YFP-R5 have been shown to express FPs by fluorescence microscopy (Figure S4). Emission maxima of the GFP and YFP are relatively close $(511 \mathrm{~nm}$ for GFPmut3 and $528 \mathrm{~nm}$ for Venus YFP), ${ }^{35,36}$ such that the same filter set is used to visualize the fluorescence of both FPs. However, fluorescence characteristics of these genetic constructs can be clearly discriminated by fluorescence spectrometry.

2.2. Silica Resin Purification of Hybrid Fluorescent Proteins. Whole cell lysates of R5 peptide-conjugated FPs were bound to the silica gel in buffer A, which was then washed to remove unbound residues and loosely attached fusion proteins. Elution was accomplished by a positively charged small molecule (1 M L-lysine in phosphate buffer) that replaces fusion proteins bound to the resin. R5-tagged FPs bind to the resin with moderate affinity $\left(k_{\mathrm{d}}=1.09 \mu \mathrm{M}\right)$ and after repeated washing steps ( $>10$ resin volume), wash fractions do not yield a qualitative fluorescence signal (Figure 1B,C). The elution of fluorescence proteins was confirmed by SDS-PAGE analysis. Gel bands corresponding to conjugate fluorescent proteins were consistent with theoretical predictions $(\approx 29 \mathrm{kDa})$ (Figure 1B, lower gel).

His-tagged FPs for each FP were also designed to compare the quality of the silica-binding tag. The proposed silica-binding tag was outperformed by commercial Hispur cobalt resin in terms of the purity calculated from total bands in the same lane. However, improvements in the target band purity were comparable between cobalt resin and silica tags (Table S4). Our data suggests that the silica-binding tag approach can be useful for further applications following protein purification. However, compared to other highly specific purification techniques, the silica tag-based approach needs to be improved if higher purity is needed. Resin retrieval yields were calculated using protein concentrations before and after the purification protocol. Yields from the Hispur cobalt resin were around 10$12 \%$ for 6x-His-tagged FPs, whereas FP-R5 constructs retrieved from the silica resin were with a yield of $15-18 \%$. Although the R5-based purification tag offers a higher purification yield, the purity of the final eluate can be improved by adjusting physicochemical conditions during protein elution. However, for our purpose, in this study, purity of the fusion proteins is good enough to induce silica formation, as can been seen from the silica particle formation assays in Figures $1 \mathrm{~B}$ and $3 \mathrm{~B}$.

2.3. Characterization of FP-R5 Fusion Proteins. The binding strength and affinity of silica nanoparticles to the R5 peptide is measured quantitatively by QCM (quartz crystal microbalance) (Figure 1D). ${ }^{37}$ Adsorption isotherms of the FP$\mathrm{R} 5$ proteins were recorded as a function of time. Correlations between the applied protein concentrations to the sensor surface with resonance frequency shifts show the strength of binding, predominantly resulting from the protein itself (Figure $1 D$, inset). Data collected from the QCM-D-binding experiments were utilized to calculate the desorption constant $\left(k_{\mathrm{d}}\right)$ of the YFP-R5 protein. The calculated desorption equilibrium constants for the constructs can be found in Table 1. However,

Table 1. Equilibrium Desorption Constant $\left(k_{\mathrm{d}}\right)$ Values of FP-R5 Proteins on the Quartz Silica Surface

$\begin{array}{lc}\text { protein fusion } & \text { equilibrium desorption constant }\left(k_{\mathrm{d}}\right)(\mu \mathrm{M}) \\ \text { YFP-R5 } & 1.09 \pm 0.4 \\ \text { GFP-R5 } & 0.73 \pm 0.43 \\ \text { mCherry-R5 } & 0.43 \pm 0.20\end{array}$

compared with the available desorption constants of designer proteins for the formation of nanoparticles in the literature, ${ }^{38}$ the values for our proteins are moderate. QCM experiments were also conducted with other R5 and His-tagged FPs (Figure S5, Table 1). Similar desorption constants were found for other R5 constructs, whereas His-tagged FP constructs resulted in a frequency change only at the highest protein concentration and desorption constants were in the millimolar range. As stated previously in the literature, proteins can control nanoparticle formation kinetics and size by interacting with precursor molecules. ${ }^{39-41}$

2.4. In Vitro Silica Synthesis with Hybrid Fluorescence Proteins. Silica nanoparticle synthesis was performed according to the protocol suggested by Betancor et al. ${ }^{42}$ with slight modifications. Before silica nanoparticle synthesis, fluorescent proteins that are encapsulated by silica (Figure 3A) exhibit similar excitation-emission maxima with unmodified FPs (Figure 2A). Excitation-emission spectra were also analyzed for His-tagged versions of FPs, excitation-emission peak wavelengths of which deviated no more than $1 \mathrm{~nm}$ from their R5-tagged counterparts. Excitation-emission pairs were expected to stay similar because the covalently attached chromophore is embedded within the barrel-shaped 3D structure $^{43}$ and the GS-linker provides spatial freedom to the fusion partners to fold properly and independently.

Some of the defining characteristics of fluorescent materials are their fluorescence decays and lifetimes, which are shown in Figure 2B. Encapsulation into silica nanoparticles did not cause any obvious change in the fluorescence lifetimes of the fusion proteins. The fluorescent lifetime values are summarized in Table 2. 
A
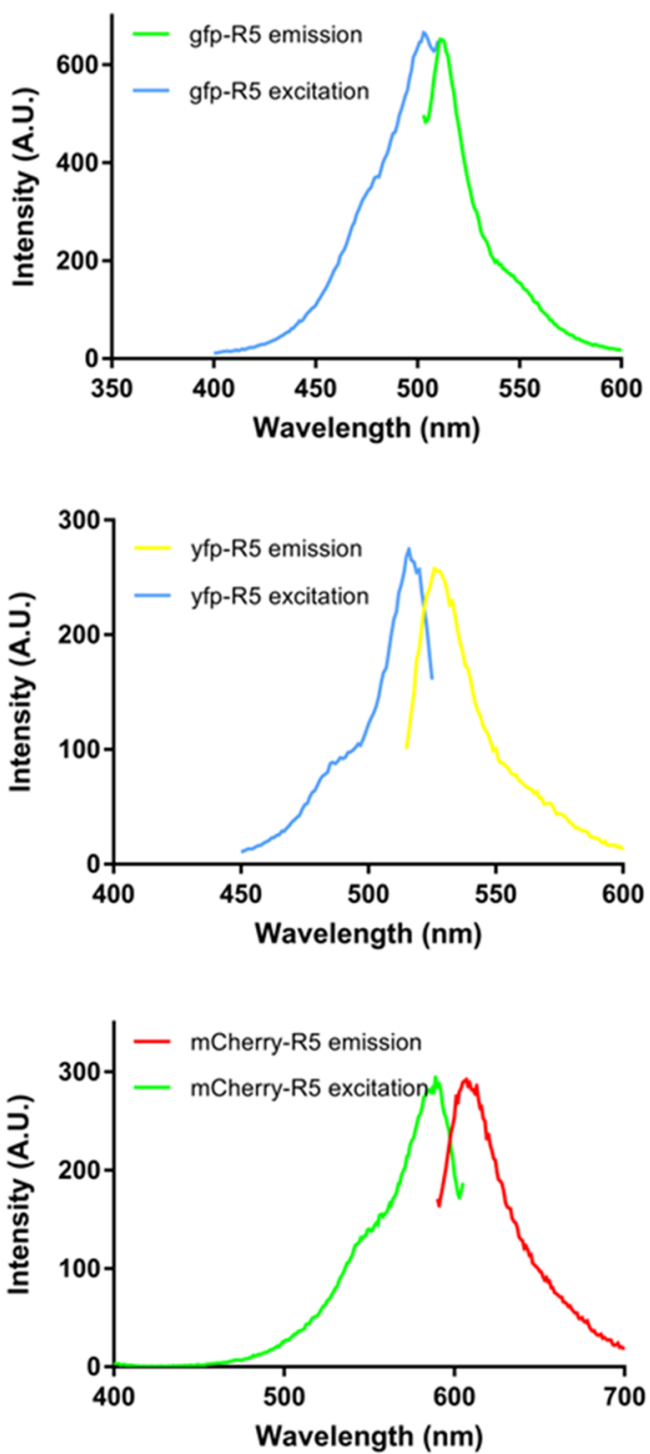

B
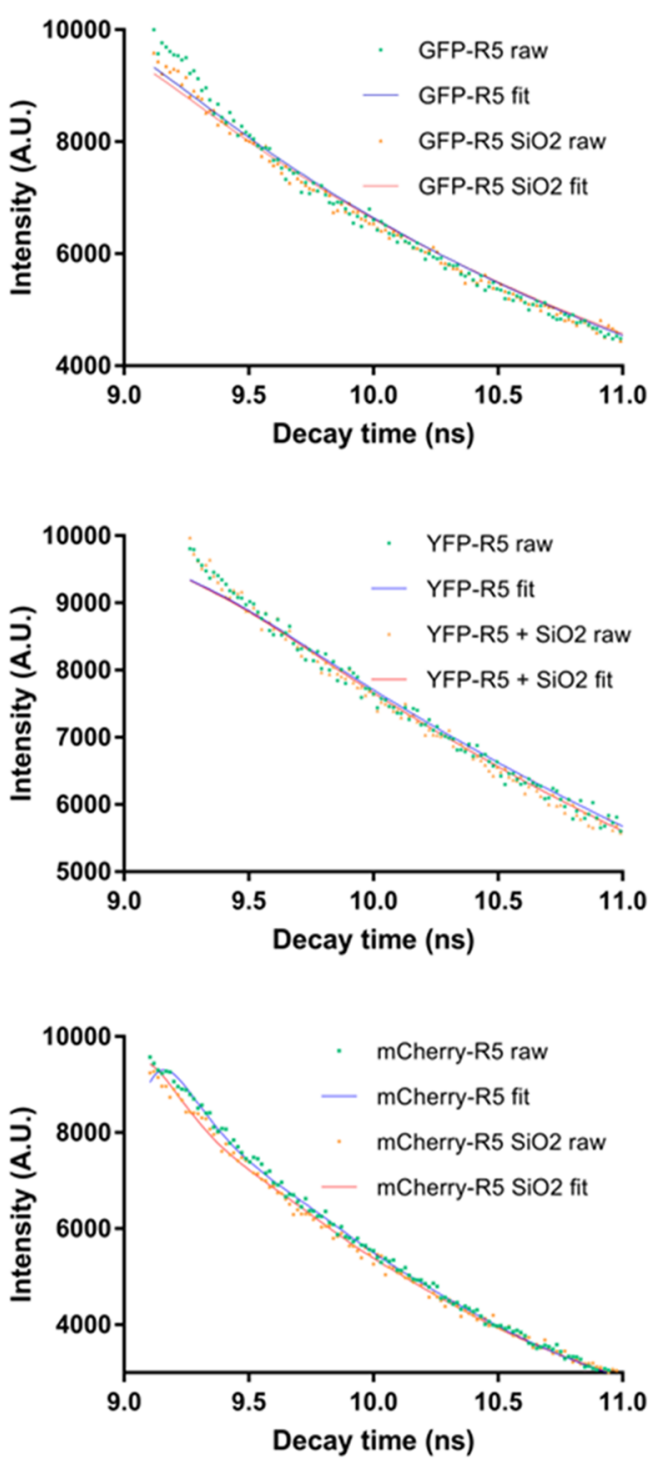

Figure 2. Fluorescence characteristics of designer proteins. Left panel (A) shows the excitation-emission spectra pairs for GFP-R5 (exc: $501 \mathrm{~nm}$, emis: $509 \mathrm{~nm}$ ), YFP-R5 (exc: $514 \mathrm{~nm}$, emis: $527 \mathrm{~nm}$ ), and mCherry-R5 (exc: $587 \mathrm{~nm}$, emis: $610 \mathrm{~nm}$ ) constructs. Right panel (B) shows timeresolved fluorescence measurements and fluorescence decay characteristics for GFP-R5, YFP-R5, and mCherry-R5 proteins before and after $\mathrm{SiO}_{2}$ encapsulation.

Table 2. Amplitude Weighted Average Fluorescence Lifetimes of Fusion Constructs

\begin{tabular}{lc}
\multicolumn{1}{c}{ fusion protein } & fluorescence lifetime (ns) \\
GFP-R5 only & 2.61 \\
GFP-R5 in $\mathrm{SiO}_{2}$ & 2.67 \\
YFP-R5 only & 3.13 \\
YFP-R5 in $\mathrm{SiO}_{2}$ & 3.06 \\
mCherry-R5 only & 0.85 \\
mCherry-R5 in $\mathrm{SiO}_{2}$ & 0.91 \\
\hline
\end{tabular}

The silica nanoparticle dispersity increases as the amount of protein added to the solution decreases, as suggested by fluorescence microscopy and SEM studies (Figures 3B and S6B). Initial parameters used in silica nanoparticle synthesis resulted in agglomerated particles of various sizes. Therefore, nanoparticle synthesis optimization was necessary for efficient synthesis. Previously, the concentration of ingredients and solution $\mathrm{pH}$ were used for the optimization of the silica nanoparticle synthesis process. ${ }^{19} \mathrm{We}$ decreased the concentration of TMOS added to the reaction mix for YFP-R5 construct to one-fourth of the initial concentration and observed that the number of freely formed silica nanoparticles, not as in aggregates, was improved as a result (details of the TMOS-based silica particle synthesis can be found in the Methods section). In addition, diluted reaction buffer was used to wash the reaction solution to remove unreacted chemicals; therefore, we have compared the single-washed reaction mixture with triple-washed samples. The quality improved by additional washing but the concentration of nanoparticles had decreased in each washing step, making additional washing steps inadvisable. Therefore, three washing steps appear to be adequate for improved quantity and yield.

Synthesized particle fluorescence was examined for all samples involved in the optimization process, which exhibited similar particle characteristics under electron microscopy. 


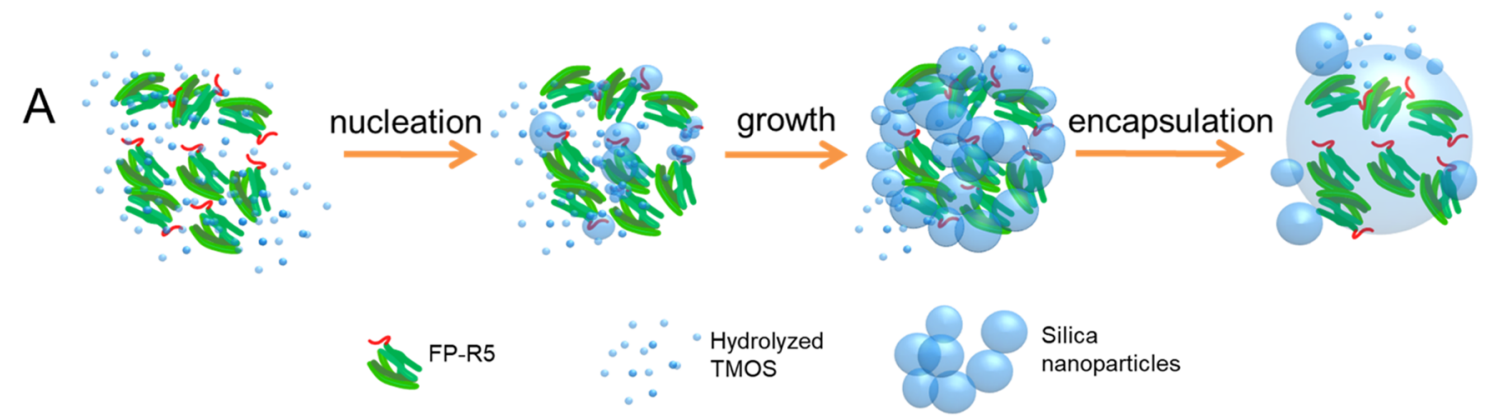

B

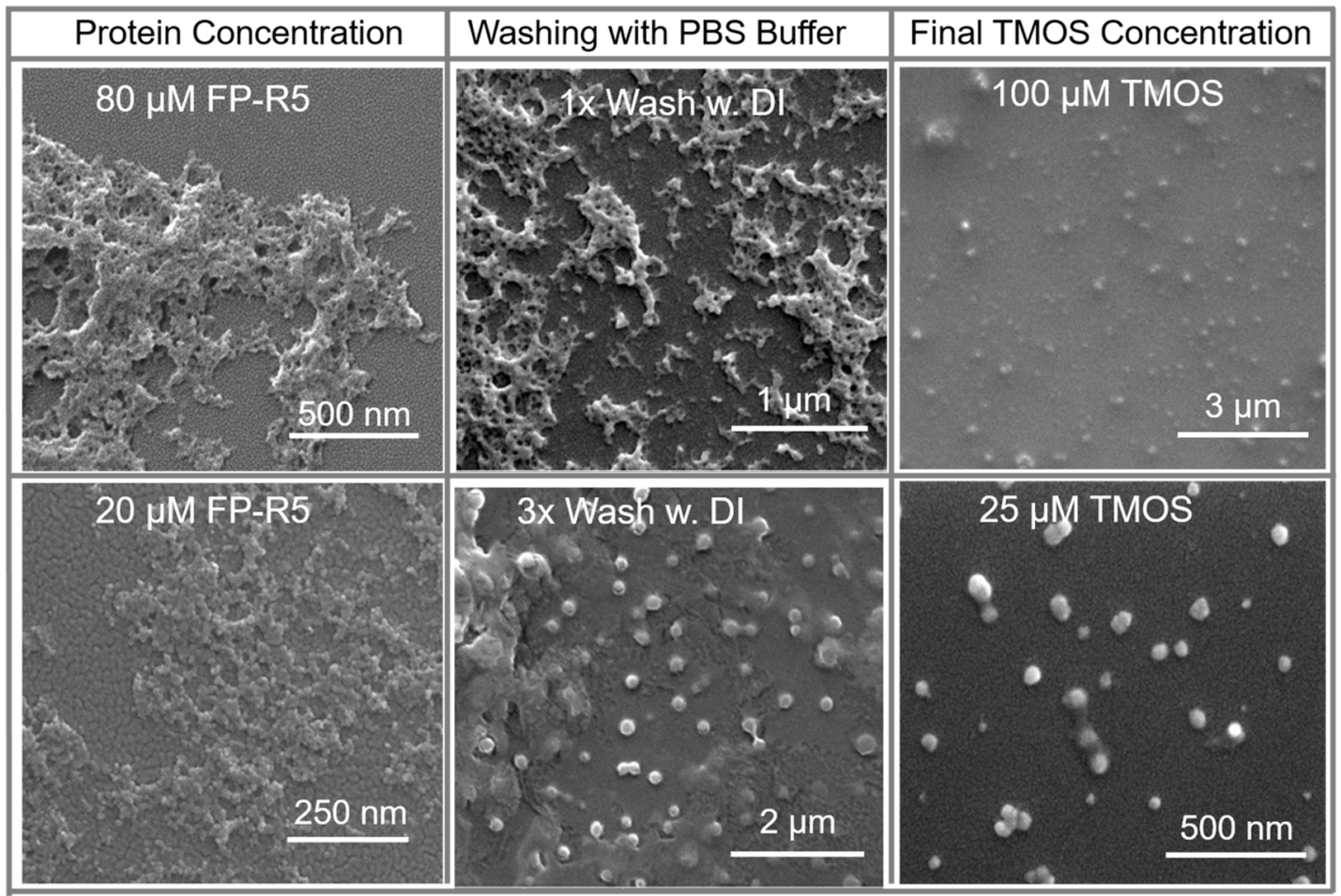

Figure 3. Silica particle synthesis characteristics. The model at (A) describes silica particle synthesis by R5 peptide conjugated to fluorescent proteins. Optimization of parameters (protein concentration, tetramethylorthosilicate (TMOS) concentration, and washing of the unreacted species) for silica nanoparticle synthesis, as shown by scanning electron microscope (SEM) imaging (B).

Higher protein concentrations during the synthesis process result in the production of larger particles (Figure S6A). Similar observations were made previously. ${ }^{44,45}$ The particle size distribution remains moderate (with a diameter of $\sim 100-200$ $\mathrm{nm}$ ) and was similar across different designer protein concentrations (Figure S7). Proteins are expected to control the growth of the nanoparticles by interacting with the precursors and modulating the nucleation process. As the particles start to grow, proteins are adsorbed on growing particles. This event prevents the attachment of the additional seeds and precursor materials, resulting in restricted growth of the final particles. ${ }^{46}$

However, in our case we observed that particles tend to grow as a function of protein concentrations. This may indicate that the proteins are encapsulated within the particles instead of attaching to particle surfaces and restricting their growth. Additional proteins induce the formation of additional layers on preformed particles, as explained in the model in Figure 3A. Fluorescence of silica particles is shown for each of the constructs in Figure S8.
The particles that are seen in the SEM and TEM images are in the nanometer range (Figures $3 \mathrm{~B}$ and 4 ). However, we may have caused the partial aggregation of the particles during sample preparation, as large structures containing nanoparticles have been observed under SEM. When FP-R5 was not added to the reaction mix, silica nanostructures were not visible (Figure S9). It is important to note that the particle shape is not perfectly spherical, and we observed a greater number of aggregates for unwashed samples: fluorescent proteins used in this study are expected to be about $4.2 \mathrm{~nm}$ in length and $2.5 \mathrm{~nm}$ in width, and surface roughness of the particles would be in proportion with these values. Silica particles also tend to form small aggregates, which may increase effective sizes. ${ }^{47-49}$

The particle origin has been shown by several methods. Energy dispersive X-ray spectroscopy (EDS) and energy filtering TEM (EFTEM) results clearly show that silicon atoms are present in the synthesized particles and atoms belonging to organic molecules $(\mathrm{C}, \mathrm{O}$, and $\mathrm{N}$ ) have likewise been observed for genetic construct-functionalized particles (Figure 4A,B). Fluorescence microscopy results support this trend (Figure S4, Supporting Information). The energy 

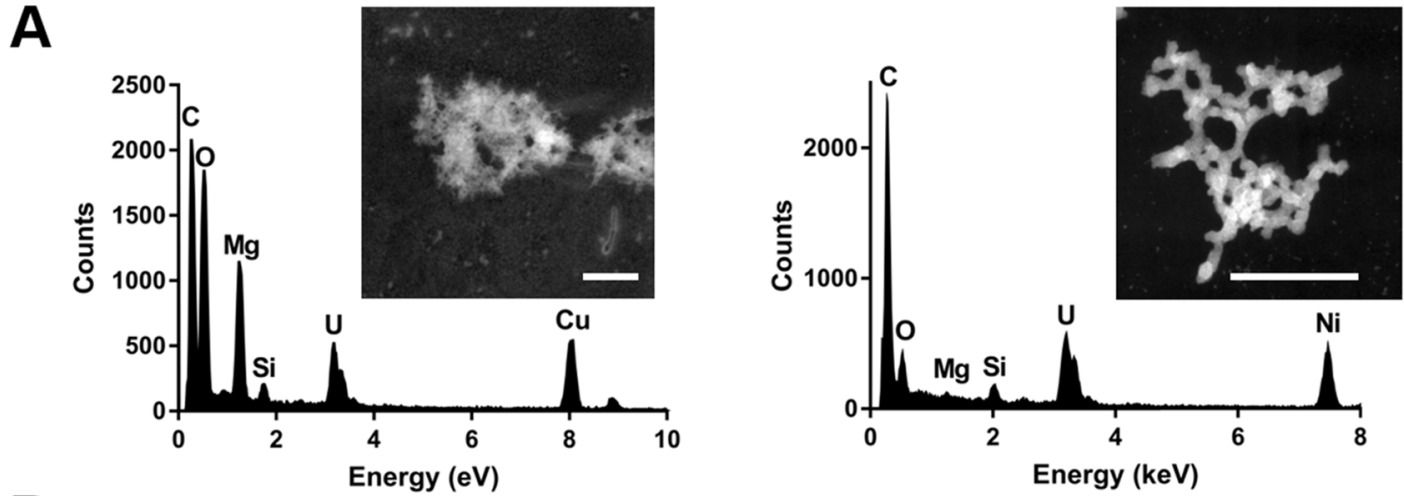

B
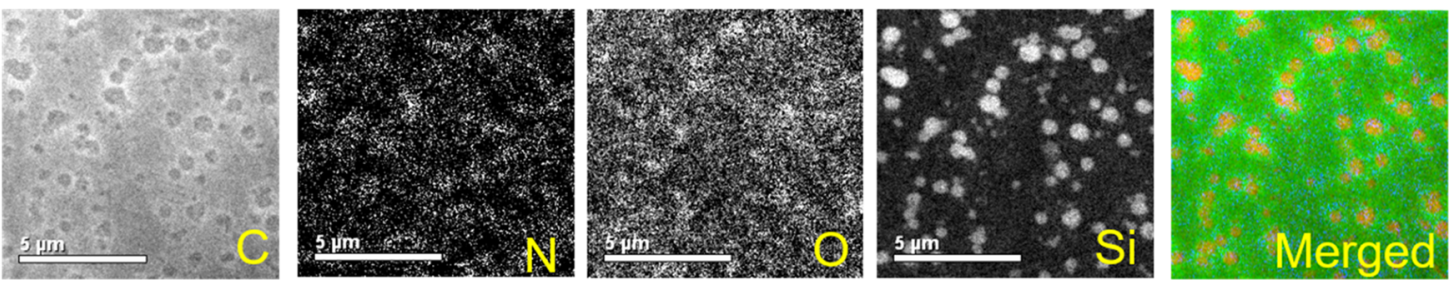

Figure 4. Transmission electron micrographs (TEM) and energy-dispersive X-ray spectroscopy (EDS) of silica containing GFP-R5 and mCherry-R5 fusion proteins, respectively (A). Graphical images show the areas where the X-ray signal was collected. White bars are of $200 \mathrm{~nm}$ length. (B) Energy-filtered TEM (EFTEM) maps of the silica containing YFP-R5 fusion proteins. C, N, O, and Si were selected for imaging. The merged figure is the sum of all signals.

spectrum shows other signal peaks that would be attributed to the composition of the grid (in the case of $\mathrm{Ni}$ and $\mathrm{Cu}$ ) or staining method that is preferred (in the case of $\mathrm{U}$ and $\mathrm{Mg}$ ).

2.5. Effects of the Silica Nanoparticles on Cell Viability. One of the application areas for biodots is biomedical imaging. In terms of imaging, cancer cells are good candidates to test. The cell viability assay was performed to check the toxicity values for the designed genetic constructs. MCF-10A is a normal human breast cell line, whereas MDAMB-231 and MDA-MB-436 are human breast adenocarcinoma cell lines. The silica concentration was measured as the total weight of the dried sample, neglecting the concentration of proteins, which are about 4 orders of magnitude less than that of silica. Results indicate that the nanoparticles do not exhibit differences between normal and cancerous cell lines in terms of cellular toxicity (Figure 5). Overall, the toxicity was comparably

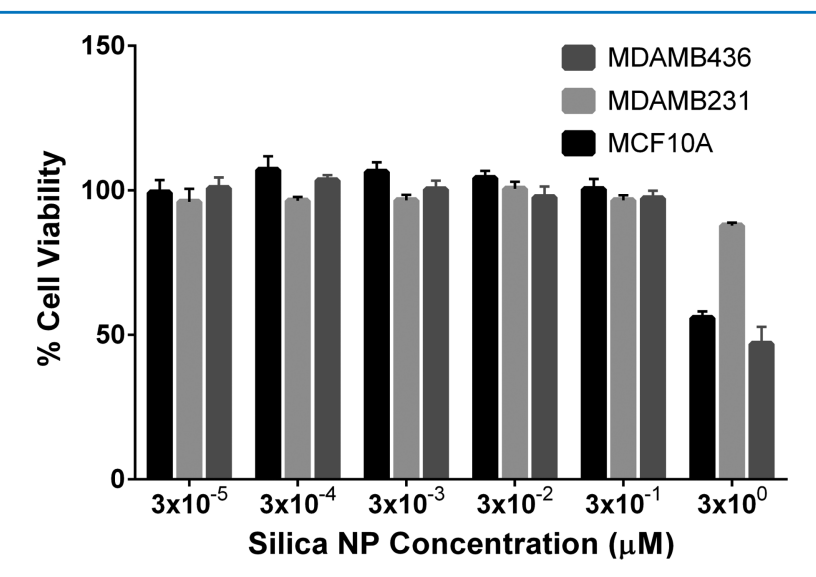

Figure 5. Effect of mCherry-R5 fluorescent silica particles (concentration range $30 \mathrm{pM}$ to $3 \mu \mathrm{M}$ ) on the proliferation of breast cancer cell lines (MDA-MB-231 and MDA-MB-436) and normal breast cell line (MCF-10A). Data represent mean \pm standard deviation $(n=4)$. low, whereas the effect of toxicity was observed both visually and enzymatically only at the highest silica concentration. Silica fluorescent particles designed in the present study are generally smaller than the particles used to study toxicity in the literature. $^{50,51}$ Contamination by a lower variety and concentration of chemicals would decrease the toxicity while decreasing size, which could significantly contribute to the reactivity of silica particles with subcellular components.

Diatom frustules can be considered as an inspiration for the development of nanosized biohybrid materials. Their ability to synthesize silica nanostructures at ambient conditions with great precision is still unsolved, but many studies prove that the degree of control in diatom silica biomineralization is intriguingly high. Coyle et al. and others have previously shown that silica-binding tags can be used as an effective protein purification tool. ${ }^{52} \mathrm{R} 5$ tag facilitates the purification of proteins without the need for other affinity tags and thereby produces significantly less impurities after purification. R5 peptide tag was used in two different operations as a bifunctional system. The first is to elute the protein of interest from the vast mixture of cellular extracts by utilizing the affinity of the tag to silica. The second is to help the synthesis of silica micro/nanostructures by nucleating hydrolyzed silica ions. It has been reported that sol-gel processing silica is a convenient material for producing fluorescent nanoparticles by the incorporation of organic dyes during production, whereas the brightness and emission of organic dye molecules can be enhanced for silica-based nanoparticles. ${ }^{53}$ During the design process of recombinant protein expression, the strategy of elution should be carefully selected. ${ }^{54}$ Bifunctional tags help in the handling and increase the speed and robustness of the whole process. Bifunctional tags are also proposed to be utilized in various applications of silica in biotechnology, such as carrier drug-gene delivery systems and biosensing. ${ }^{55,56}$ Fluorescent silica biodots can be utilized in biomedical applications not only for imaging purposes but also can be designed as a drug and 
gene carried with real-time monitoring capabilities. Given the wide range applicability and biocompatibility of silica nano/ microparticles, biodots can find a wide range of applicability. The protein-based nano/microparticle synthesis is a promising approach in terms of lower toxicity, ease of tunability, and modularity.

\section{METHODS}

3.1. Cloning of Fluorescent Proteins. GFP-R5 and R5GFP fusion cassettes were produced by four successive PCRs using GFPmut3 as a template. R5 peptide $\left(\mathrm{H}_{2} \mathrm{~N}\right.$ SSKKSGSYSGSKGSKRRIL- $\mathrm{CO}_{2} \mathrm{H}$ ) coding DNA sequence is added to the $3^{\prime}$ and $5^{\prime}$ end of the FP coding DNA sequences. GS flexible linkers are motifs of glycine and serine that are used in varying numbers and allow the separated folding of adjacent parts. Three repeats of GGGS were inserted between the FPs and R5. PCR was performed using Q5 DNA polymerase (NEB), and the reaction mix was prepared according to the manufacturer's protocol. Primers are listed in Table S1 in the Supporting Information. $T_{\mathrm{m}}$ (melting temperature) was calculated using IDT oligo analyzer software. The PCR reaction setup is as follows: initial denaturation $\left(98{ }^{\circ} \mathrm{C}, 30\right.$ $\mathrm{s}), 5$ cycles of amplification for the primer-binding region of DNA through denaturation $\left(98^{\circ} \mathrm{C}, 10 \mathrm{~s}\right)$, primer annealing (binding $\left.T_{\mathrm{m}}, 30 \mathrm{~s}\right)$ and extension $\left(72{ }^{\circ} \mathrm{C}, 30 \mathrm{~s} / \mathrm{kb}\right), 30$ cycles of amplification for the total primer length through denaturation $\left(98{ }^{\circ} \mathrm{C}, 10 \mathrm{~s}\right)$, primer annealing (total $\left.T_{\mathrm{m}}, 30 \mathrm{~s}\right)$ and extension $\left(72{ }^{\circ} \mathrm{C}, 30 \mathrm{~s} / \mathrm{kb}\right)$, and final extension for $5 \mathrm{~min}$. The resulting amplicon and the vector were KpnI and MluI (NEB)-digested; gel was extracted and ligated by T4 DNA ligase (NEB) according to the manufacturer's protocol.

To produce YFP-R5 and mCherry-R5 cassettes, E. coli codon-optimized R5 peptide-coding sequence was artificially synthesized (Sentegen, Turkey). Each genetic part was then PCR-amplified to include homologous regions. These parts were then ligated into an anhydrotetracycline ( $\mathrm{aTc}$ )-inducible pZA vector using the Gibson Assembly protocol. ${ }^{57}$ To amplify the DNA sequence of the YFP and mCherry, pZS2-123 plasmid was utilized (Addgene \#26598). ${ }^{58}$

Ligated plasmid constructs were then transformed into chemically competent E. coli DH5 $\alpha$ PRO strain by the standard heat-shock transformation protocol. Colonies growing on antibiotic agar plates supplemented with chloramphenicol (35 $\mu \mathrm{g} / \mathrm{mL}$ ) were chosen, and all FP-R5 constructs were verified by DNA sequencing (Figure S2). Amino acid sequences of proteins and nucleotide sequences of DNA are listed in Table S2.

3.2. Overexpression of the Fusion Proteins. Following DNA sequence verification, $E$. coli DH5 $\alpha$ PRO strains that contain FP-R5 expression constructs were grown in low-salt lysogeny broth medium (tryptone $10 \mathrm{~g} / \mathrm{L}, \mathrm{NaCl} 5 \mathrm{~g} / \mathrm{L}$, yeast extract $5 \mathrm{~g} / \mathrm{L}$ ) supplemented with chloramphenicol. At an OD600 of 0.6 , aTc $(100 \mathrm{ng} / \mathrm{mL})$ was added to each bacterial culture. Following induction, the cultures were incubated for 8 $\mathrm{h}$ in a $30^{\circ} \mathrm{C}\left(20^{\circ} \mathrm{C}\right.$ for mCherry-R5) shaker at $200 \mathrm{rpm}$ and the cultures were then harvested by centrifugation at $5000 \mathrm{~g}$ for $15 \mathrm{~min}$ at $4{ }^{\circ} \mathrm{C}$ and suspended in extraction buffer $(20 \mathrm{mM}$ Tris, $2 \mathrm{mM}$ ethylenediaminetetraacetic acid, $\mathrm{pH}$ : 7.5). Total protein extraction was performed by probe sonication for $5 \mathrm{~min}$ (10/10 s on/off regime). Then, extracts were centrifuged at 15 $000 \mathrm{~g}$ for $1 \mathrm{~h}$ and the supernatant was retrieved for later use.

3.3. Purification of Tagged Proteins via Silica Resin. The purification of R5-tagged proteins was performed according to the protocol by Coyle et al., ${ }^{52}$ with modifications. The silica gel matrix was prepared by washing silica microspheres (Sigma) having the sizes of $60-220 \mathrm{~mm}$ with protein extraction buffer. Protein extracts were incubated with the silica gel overnight at $4{ }^{\circ} \mathrm{C}$ while being agitated, and the gel was then washed several times with $10 \mathrm{mM}$ L-lysine solution in protein extraction buffer to remove excess R5-FPs and unbound solutes. Elution was performed by adding $1 \mathrm{M} \mathrm{L}$ lysine containing $2 \%$ glacial acetic acid to the silica gel/total protein mixture. Elution fractions were collected for SDSPAGE gel analysis. Purified protein concentrations were determined by bichinconinic acid assay (BCA, Pierce). Bifunctional protein molecular weights and $\mathrm{pI}$ values were calculated using ExPASy online tool (Table S3). 6x-His tagged experimental control samples were purified by Hispur cobalt resin (Thermo Scientific). Purified proteins were visualized by 10\% TGX SDS-PAGE using Mini-Protean Handcast System (Biorad). The purity of SDS-PAGE bands corresponding to the fusion proteins were calculated by quantification of protein bands by Image J program. Fold change values were calculated as a ratio of purity of the target band in the same lane, before and after the purification.

3.4. Silica Nanoparticle Synthesis. Chemical synthesis of silica nanoparticles is generally achieved by modified versions of the Stöber process. ${ }^{42}$ In this study, tetramethylorthosilicate (TMOS) was used as the silica precursor and prepared as $1 \mathrm{M}$ in $\mathrm{ddH}_{2} \mathrm{O}$ and $1 \mathrm{mM} \mathrm{HCl}$ is added to hydrolyze the TMOS by stirring. Twenty five microliters of $200 \mu \mathrm{M}$ eluted proteins are added to $0.1 \mathrm{M}$ phosphate citrate buffer ( $\mathrm{pH}: 8)$, and $50 \mu \mathrm{L}$ of $1 \mathrm{M}$ TMOS is added slowly into the reaction solution to bring the total volume to $1 \mathrm{~mL}$. Then, resulting mixtures are vortexed and washed several times with $0.025 \mathrm{M}$ phosphate citrate buffer $(\mathrm{pH}: 7.5)$ to remove unreacted ingredients. Resulting solutions are then probe-sonicated for 5-10 $\mathrm{min}(10 / 10 \mathrm{~s}$ on/off regime). Polyethyleneimine (10\%) was used as a positive control.

FP-R5 protein-mediated silica nanoparticle synthesis is optimized by changing the concentration of proteins used to induce the initialization of silica nanoparticle formation. Serial dilutions of the proteins are used to determine the optimal parameters for the successful synthesis of FP-containing silica nanomaterial.

Concentrations of the ingredients were optimized for efficient and stable synthesis of the nanomaterials. Nanomaterial synthesis protocols generally suggest addition of ingredients at low rates usually by help of a peristaltic pump. In our case, we added TMOS in five equal volume shots $(5 \times$ $10 \mu \mathrm{L})$ to increase particle quality. ${ }^{59}$

3.5. Fluorescence Measurements. To detect potential shifts in the peak values or emergence of new peaks of fluorescence excitations and emissions of purified recombinant fluorescent proteins, iterative excitation and emission scanning is performed. Spectral data has been collected for those optimized wavelengths using fluorescence spectrometry (Agilent Cary Eclipse).

Samples were prepared on glass slides for fluorescence microscopy imaging. GFP-R5, R5-GFP, and YFP-R5 were visualized using fluorescence microscopy (Scope A1 Zeiss, Germany). Approximately $10-15 \mu \mathrm{L}$ of samples were dropped on a microscopy glass slide and covered with a coverslip. Filter sets were chosen according to the excitation-emission pairs for each of the FPs. fs 20 is selected for detecting red fluorescence, whereas fs 38 was chosen for green and yellow fluorescence. 
Fluorescence lifetimes and decay kinetics were measured by time-resolved fluorescence analysis (Pico-quant, Germany). Emission wavelengths of FPs were previously determined by fluorescence spectrometry data. Fluorescent decay tail fit was performed by Fluofit software.

3.6. Quartz Crystal Microbalance Experiments. Quartz crystal microbalance with dissipation monitoring (QCM-D) quartz sensors were purchased from Biolin Scientific, Sweden. The sensors were cleaned by UV-ozone and 2\% SDS treatment according to the manufacturer's protocol. Sensor surfaces were prewashed with $1 \times$ PBS for $1 \mathrm{~h}$. Protein samples were prepared in $1 \times$ PBS solution at concentrations of 100 , $200,500,1000$, and $2000 \mathrm{nM}$ and administered from the lowest to highest concentration. After each protein administration, $1 \times$ PBS washing was performed until frequency change values reached a plateau. Measurements were performed in the Qsense QCM explorer equipment. The data selected for calculations was from three consecutive overtone orders $n=$ 3, 5, and 7. The Langmuir equilibrium model was utilized to estimate the adsorption characteristics of the FPs to the silica sensor surface. In this model, frequency change is related to the desorption strength by the equation $\Delta f=\left(f_{\max } \times C\right) /\left(k_{\mathrm{d}}+C\right)$, where $C$ is the concentration of the protein. $f_{\max }$ value was estimated by the Langmuir isotherm, and $k_{\mathrm{d}}$ value was determined by least squares fitting accordingly. ${ }^{38}$

3.7. Cellular Toxicity of Fluorescent Silica Particles. MDA-MB-231, MDA-MB-436, and MCF-10A cell lines were obtained from the American Type Culture Collection (Manassas, VA). Cells were cultured in $100 \mathrm{~mm}$ Petri dishes at $37{ }^{\circ} \mathrm{C}$ and $5 \% \mathrm{CO}_{2}$. Dulbecco's modified Eagle medium with $10 \%$ fetal bovine serum and $1 \%$ nonessential amino acids was used for culturing of the cells. To examine the effect of silica particles on cell viability, cells were seeded (6000 cells/well) in 96-well plates with $100 \mu \mathrm{L}$ of media. Silica particle treatment was performed $24 \mathrm{~h}$ after seeding. Five silica particle concentrations were prepared with serial dilutions (10-fold per dilution) for toxicity experiments. Four replicates were used for each condition. Following treatment, cells were incubated for $72 \mathrm{~h}$ at $37^{\circ} \mathrm{C}$ and $5 \% \mathrm{CO}_{2}$. CellTiter-Glo Cell Viability Kit was used to examine the effect of the particles on cell viability. At the end of the $72 \mathrm{~h}$ silica particle incubation period, cells were removed from the incubator and incubated at room temperature along with CellTiter-Glo reagent for $30 \mathrm{~min}$. After reaching the temperature equilibrium, CellTiter-Glo reagent was administered to each well in a volume of $30 \mu \mathrm{L}$. Then, the plate was placed on a shaker for $10 \mathrm{~min}$ to facilitate cell lysis. Finally, medium-reagent mixture from each 96-well plate was transferred to corresponding wells of opaque white flat bottom 96-well plates, and the luminescence signal was measured using Biotek Microplate reader.

3.8. Scanning Electron Microscopy. SEM studies were performed either with unmodified silicon wafers or $40 \mu \mathrm{m}$ of gold deposited by thermal evaporation on glass substrates. A microliter of the $1 \times$ PBS-washed sample was drop cast on the surface and air-dried for $30 \mathrm{~min}$. Samples were then sputtercoated with gold-palladium to obtain small grain sizes for highresolution images. The thin-film coating thickness was around $3-5 \mathrm{~nm}$. Thin-film coating significantly reduces electron energy absorption-related burns (charging) on the sample. SEM (FEI Quanta) images were taken at varying forward voltages for electron energy $(5-20 \mathrm{keV})$.

3.9. Transmission Electron Microscopy. TEM studies were conducted to visualize the particles at a higher magnification. Fifteen microliters of samples were put on a parafilm surface, and an EMS Ni grid with formvar/C support (300 mesh) was placed on the sample with the carbon-coated surface facing the sample. After $1 \mathrm{~min}$ of incubation, excess fluid that is adhered to the surface was removed by letting the side of the grid touch a tissue paper. Three rounds of distilled deionized water cleaning were performed. Samples were stained with $2 \%$ uranyl acetate for $30 \mathrm{~s}$ to improve the image contrast. The samples were then analyzed on TEM (FEI Tecnai). TEM electron acceleration power was $200 \mathrm{keV}$. Energy dispersive Xray spectroscopy (EDS) analysis was also performed to identify material composition (EDAX).

\section{ASSOCIATED CONTENT}

\section{Supporting Information}

The Supporting Information is available free of charge on the ACS Publications website at DOI: 10.1021/acsomega.7b01769.

Primers used, DNA and amino acid sequences of constructs, $M_{\mathrm{w}}$ and $\mathrm{pI}$ of FPs, fluorescence spectra of R5-GFP, Sanger sequencing results, fluorescence microscopy images, purity of SDS-PAGE bands, quartz crystal microbalance (QCM) measurements, silica particle size distribution graph, fluorescence images of FP concentration-dependent silica synthesis, bright field and fluorescence microscopy images of silica particles; SEM of the synthesis product when FP-R5 is absent (PDF)

\section{AUTHOR INFORMATION}

\section{Corresponding Author}

*E-mail: urartu@bilkent.edu.tr.

ORCID $\odot$

Urartu Ozgur Safak Seker: 0000-0002-5272-1876

Notes

The authors declare no competing financial interest.

\section{ACKNOWLEDGMENTS}

The study was supported by TUBİTAK Project 115M108. U.O.S.S. is grateful to TUBA-GEBIP (Turkish Academy of Sciences Young Investigator Award), Science Academy Award (BAGEP), and FABED Award and T.T.O. is grateful to TUBITAK-BIDEB Graduate Scholarship. Authors thank Michael Elowitz for the pZS2-123 plasmid via Addgene. We also thank Prof. Hilmi Volkan Demir for allowing us to use Time Resolved Fluorescence Spectroscopy and Quartz Crystal Microbalance devices.

\section{NOMENCLATURE}

EDS, energy-dispersive X-ray spectroscopy; EFTEM, energy filtering transmission electron microscope; GFP, green fluorescent protein; LB, lysogeny broth; PEI, polyethyleneimide; QCM, quartz crystal microbalance; SDS-PAGE, sodium dodecyl sulfate-polyacrylamide gel electrophoresis; SEM, scanning electron microscope; TEM, transmission electron microscope; TMOS, tetramethylorthosilicate; YFP, yellow fluorescent protein

\section{REFERENCES}

(1) Hildebrand, M. Diatoms, biomineralization processes, and genomics. Chem. Rev. 2008, 108, 4855-4874.

(2) Weiner, S.; Dove, P. M. An overview of biomineralization processes and the problem of the vital effect. Rev. Mineral. Geochem. 2003, 54, 1-29. 
(3) Chen, P.-Y.; Lin, A. Y.; Lin, Y. S.; Seki, Y.; Stokes, A. G.; Peyras, J.; Olevsky, E. A.; Meyers, M. A.; McKittrick, J. Structure and mechanical properties of selected biological materials. J. Mech. Behav. Biomed. Mater. 2008, 1, 208-226.

(4) Bègue-Kirn, C.; Krebsbach, P. H.; Bartlett, J. D.; Butler, W. T. Dentin sialoprotein, dentin phosphoprotein, enamelysin and ameloblastin: tooth-specific molecules that are distinctively expressed during murine dental differentiation. Eur. J. Oral Sci. 1998, 106, 963-970.

(5) Gajjeraman, S.; Narayanan, K.; Hao, J.; Qin, C.; George, A. Matrix macromolecules in hard tissues control the nucleation and hierarchical assembly of hydroxyapatite. J. Biol. Chem. 2007, 282, 1193-1204.

(6) D’Souza, R. N.; Cavender, A.; Sunavala, G.; Alvarez, J.; Ohshima, T.; Kulkarni, A. B.; MacDougall, M. Gene expression patterns of murine dentin matrix protein 1 (Dmp1) and dentin sialophosphoprotein (DSPP) suggest distinct developmental functions in vivo. J. Bone Miner. Res. 1997, 12, 2040-2049.

(7) Falini, G.; Albeck, S.; Weiner, S.; Addadi, L. Control of aragonite or calcite polymorphism by Mollusk shell macromolecules. Science 1996, 271, 67-69.

(8) Zhang, B.; Wustman, B. A.; Morse, D.; Evans, J. S. Model peptide studies of sequence regions in the elastomeric biomineralization protein, Lustrin A. I. The C-domain consensus-PG-, -NVNCT-motif. Biopolymers 2002, 63, 358-369.

(9) Armbrust, E. V. The life of diatoms in the world's oceans. Nature 2009, 459, 185-192.

(10) Ferrara, M. A.; Dardano, P.; De Stefano, L.; Rea, I.; Coppola, G.; Rendina, I.; Congestri, R.; Antonucci, A.; De Stefano, M.; De Tommasi, E. Optical properties of diatom nanostructured biosilica in Arachnoidiscus sp: micro-optics from mother nature. PLoS One 2014, 9, No. e103750.

(11) Rao, K. S.; El-Hami, K.; Kodaki, T.; Matsushige, K.; Makino, K. A novel method for synthesis of silica nanoparticles. J. Colloid Interface Sci. 2005, 289, 125-131.

(12) Wang, Y.; Xia, Y. N. Bottom-up and top-down approaches to the synthesis of monodispersed spherical colloids of low melting-point metals. Nano Lett. 2004, 4, 2047-2050.

(13) Hussain, I.; Singh, N. B.; Singh, A.; Singh, H.; Singh, S. C. Green synthesis of nanoparticles and its potential application. Biotechnol. Lett. 2016, 38, 545-560.

(14) Thakkar, K. N.; Mhatre, S. S.; Parikh, R. Y. Biological synthesis of metallic nanoparticles. Nanomedicine 2010, 6, 257-262.

(15) Nassif, N.; Livage, J. From diatoms to silica-based biohybrids. Chem. Soc. Rev. 2011, 40, 849-859.

(16) Cui, R.; Liu, H. H.; Xie, H. Y.; Zhang, Z. L.; Yang, Y. R.; Pang, D. W.; Xie, Z. X.; Chen, B. B.; Hu, B.; Shen, P. Living yeast cells as a controllable biosynthesizer for fluorescent quantum dots. Adv. Funct. Mater. 2009, 19, 2359-2364.

(17) Scheffel, A.; Poulsen, N.; Shian, S.; Kroger, N. Nanopatterned protein microrings from a diatom that direct silica morphogenesis. Proc. Natl. Acad. Sci. U.S.A. 2011, 108, 3175-3180.

(18) Shrestha, R. P.; Hildebrand, M. Evidence for a regulatory role of diatom silicon transporters in cellular silicon responses. Eukaryotic Cell 2015, 14, 29-40.

(19) Senior, L.; Crump, M. P.; Williams, C.; Booth, P. J.; Mann, S.; Perriman, A. W.; Curnow, P. Structure and function of the silicifying peptide R5. J. Mater. Chem. B 2015, 3, 2607-2614.

(20) Lechner, C. C.; Becker, C. F. Modified silaffin R5 peptides enable encapsulation and release of cargo molecules from biomimetic silica particles. Bioorg. Med. Chem. 2013, 21, 3533-3541.

(21) Knecht, M. R.; Wright, D. W. Functional analysis of the biomimetic silica precipitating activity of the R5 peptide from Cylindrotheca fusiformis. Chem. Commun. 2003, 3038-3039.

(22) Naik, R. R.; Whitlock, P. W.; Rodriguez, F.; Brott, L. L.; Glawe, D. D.; Clarson, S. J.; Stone, M. O. Controlled formation of biosilica structures in vitro. Chem. Commun. 2003, 238-239.

(23) Naik, R. R; Tomczak, M. M.; Luckarift, H. R.; Spain, J. C.; Stone, M. O. Entrapment of enzymes and nanoparticles using biomimetically synthesized silica. Chem. Commun. 2004, 1684-1685.
(24) Luckarift, H. R.; Spain, J. C.; Naik, R. R.; Stone, M. O. Enzyme immobilization in a biomimetic silica support. Nat. Biotechnol. 2004, 22, 211-213.

(25) Marner, W. D., 2nd; Shaikh, A. S.; Muller, S. J.; Keasling, J. D. Enzyme immobilization via silaffin-mediated autoencapsulation in a biosilica support. Biotechnol. Prog. 2009, 25, 417-423.

(26) Nam, D. H.; Won, K.; Kim, Y. H.; Sang, B. I. A novel route for immobilization of proteins to silica particles incorporating silaffin domains. Biotechnol. Prog. 2009, 25, 1643-1649.

(27) Roberts, J. R.; Antonini, J. M.; Porter, D. W.; Chapman, R. S.; Scabilloni, J. F.; Young, S. H.; Schwegler-Berry, D.; Castranova, V.; Mercer, R. R. Lung toxicity and biodistribution of Cd/Se-ZnS quantum dots with different surface functional groups after pulmonary exposure in rats. Part. Fibre Toxicol. 2013, 10, 5.

(28) Valizadeh, A.; Mikaeili, H.; Samiei, M.; Farkhani, S. M.; Zarghami, N.; Kouhi, M.; Akbarzadeh, A.; Davaran, S. Quantum dots: synthesis, bioapplications, and toxicity. Nanoscale Res. Lett. 2012, 7, No. 480.

(29) Lichty, J. J.; Malecki, J. L.; Agnew, H. D.; Michelson-Horowitz, D. J.; Tan, S. Comparison of affinity tags for protein purification. Protein Expression Purif. 2005, 41, 98-105.

(30) Abdelhamid, M. A. A.; Motomura, K.; Ikeda, T.; Ishida, T.; Hirota, R.; Kuroda, A. Affinity purification of recombinant proteins using a novel silica-binding peptide as a fusion tag. Appl. Microbiol. Biotechnol. 2014, 98, 5677-5684.

(31) Ikeda, T.; Ninomiya, K.-I.; Hirota, R.; Kuroda, A. Single-step affinity purification of recombinant proteins using the silica-binding $\mathrm{Si}$ tag as a fusion partner. Protein Expression Purif. 2010, 71, 91-95.

(32) Taniguchi, K.; Nomura, K.; Hata, Y.; Nishimura, T.; Asami, Y.; Kuroda, A. The Si-tag for immobilizing proteins on a silica surface. Biotechnol. Bioeng. 2007, 96, 1023-1029.

(33) Terpe, K. Overview of tag protein fusions: from molecular and biochemical fundamentals to commercial systems. Appl. Microbiol. Biotechnol. 2003, 60, 523-533.

(34) Isaacs, F. J.; Dwyer, D. J.; Ding, C.; Pervouchine, D. D.; Cantor, C. R.; Collins, J. J. Engineered riboregulators enable post-transcriptional control of gene expression. Nat. Biotechnol. 2004, 22, 841-847.

(35) Cowan, S. E.; Gilbert, E.; Khlebnikov, A.; Keasling, J. D. Dual labeling with green fluorescent proteins for confocal microscopy. Appl. Environ. Microbiol. 2000, 66, 413-418.

(36) Nagai, T.; Ibata, K.; Park, E. S.; Kubota, M.; Mikoshiba, K.; Miyawaki, A. A variant of yellow fluorescent protein with fast and efficient maturation for cell-biological applications. Nat. Biotechnol. 2002, 20, 87-90.

(37) Li, L.; Mu, Q. X.; Zhang, B.; Yan, B. Analytical strategies for detecting nanoparticle-protein interactions. Analyst 2010, 135, 15191530.

(38) Yuca, E.; Karatas, A. Y.; Seker, U. O.; Gungormus, M.; DinlerDoganay, G.; Sarikaya, M.; Tamerler, C. In vitro labeling of hydroxyapatite minerals by an engineered protein. Biotechnol. Bioeng. 2011, 108, 1021-1030.

(39) Jun, J. Y.; Hoang, H. N.; Paik, S. Y. R.; Chun, H. S.; Kang, B. C.; Ko, S. Preparation of size-controlled bovine serum albumin (BSA) nanoparticles by a modified desolvation method. Food Chem. 2011, 127, $1892-1898$.

(40) Leng, Y.; Fu, L.; Ye, L.; Li, B.; Xu, X.; Xing, X.; He, J.; Song, Y.; Leng, C.; Guo, Y.; Ji, X.; Lu, Z. Protein-directed synthesis of highly monodispersed, spherical gold nanoparticles and their applications in multidimensional sensing. Sci. Rep. 2016, 6, No. 28900.

(41) Wei, H.; Wang, Z.; Zhang, J.; House, S.; Gao, Y. G.; Yang, L.; Robinson, H.; Tan, L. H.; Xing, H.; Hou, C.; Robertson, I. M.; Zuo, J. M.; Lu, Y. Time-dependent, protein-directed growth of gold nanoparticles within a single crystal of lysozyme. Nat. Nanotechnol. 2011, 6, 93-97.

(42) Jackson, E.; Ferrari, M.; Cuestas-Ayllon, C.; Fernandez-Pacheco, R; Perez-Carvajal, J.; de la Fuente, J. M.; Grazu, V.; Betancor, L. Protein-templated biomimetic silica nanoparticles. Langmuir 2015, 31, 3687-3695. 
(43) Reid, B. G.; Flynn, G. C. Chromophore formation in green fluorescent protein. Biochemistry 1997, 36, 6786-6791.

(44) Lee, K. T.; Sathyagal, A. N.; McCormick, A. V. A closer look at an aggregation model of the Stober process. Colloids Surf., A 1998, $144,115-125$.

(45) Rahman, I. A.; Vejayakumaran, P.; Sipaut, C. S.; Ismail, J.; Abu Bakar, M.; Adnan, R.; Chee, C. K. An optimized sol-gel synthesis of stable primary equivalent silica particles. Colloids Surf., A 2007, 294, $102-110$.

(46) Wei, H.; Wang, Z. D.; Zhang, J.; House, S.; Gao, Y. G.; Yang, L. M.; Robinson, H.; Tan, L. H.; Xing, H.; Hou, C. J.; Robertson, I. M.; Zuo, J. M.; Lu, Y. Time-dependent, protein-directed growth of gold nanoparticles within a single crystal of lysozyme. Nat. Nanotechnol. 2011, 6, 93-97.

(47) Liu, L. Aggregation of silica nanoparticles in an aqueous suspension. AIChE J. 2015, 61, 2136-2146.

(48) Kobayashi, M.; Juillerat, F.; Galletto, P.; Bowen, P.; Borkovec, M. Aggregation and charging of colloidal silica particles: effect of particle size. Langmuir 2005, 21, 5761-5769.

(49) Bagwe, R. P.; Hilliard, L. R.; Tan, W. H. Surface modification of silica nanoparticles to reduce aggregation and nonspecific binding. Langmuir 2006, 22, 4357-4362.

(50) Lin, W.; Huang, Y. W.; Zhou, X. D.; Ma, Y. In vitro toxicity of silica nanoparticles in human lung cancer cells. Toxicol. Appl. Pharmacol. 2006, 217, 252-259.

(51) Kim, I. Y.; Joachim, E.; Choi, H.; Kim, K. Toxicity of silica nanoparticles depends on size, dose, and cell type. Nanomedicine 2015, 11, 1407-1416.

(52) Coyle, B. L.; Baneyx, F. A cleavable silica-binding affinity tag for rapid and inexpensive protein purification. Biotechnol. Bioeng. 2014, 111, 2019-2026.

(53) Burns, A.; Ow, H.; Wiesner, U. Fluorescent core-shell silica nanoparticles: towards "Lab on a Particle" architectures for nanobiotechnology. Chem. Soc. Rev. 2006, 35, 1028-1042.

(54) Arnau, J.; Lauritzen, C.; Petersen, G. E.; Pedersen, J. Current strategies for the use of affinity tags and tag removal for the purification of recombinant proteins. Protein Expression Purif. 2006, 48, $1-13$.

(55) Allison, D. P.; Dufrene, Y. F.; Doktycz, M. J.; Hildebrand, M. Biomineralization at the nanoscale learning from diatoms. Methods Cell Biol. 2008, 90, 61-86.

(56) Dolatabadi, J. E. N.; de la Guardia, M. Applications of diatoms and silica nanotechnology in biosensing, drug and gene delivery, and formation of complex metal nanostructures. TrAC, Trends Anal. Chem. 2011, 30, 1538-1548.

(57) Gibson, D. G.; Young, L.; Chuang, R. Y.; Venter, J. C.; Hutchison, C. A.; Smith, H. O. Enzymatic assembly of DNA molecules up to several hundred kilobases. Nat. Methods 2009, 6, 343-345.

(58) Cox, R. S., 3rd; Dunlop, M. J.; Elowitz, M. B. A synthetic threecolor scaffold for monitoring genetic regulation and noise. J. Biol. Eng. 2010, 4, 10.

(59) Shimogaki, T.; Tokoro, H.; Tabuchi, M.; Koike, N.; Yamashina, Y.; Takahashi, M. Large-scale synthesis of monodisperse microporous silica nanoparticles by gradual injection of reactants. J. Sol-Gel Sci. Technol. 2015, 74, 109-113. 